\title{
Family planning practice among Christian health service providers in Ghana: a case study
}

\section{James Duah ${ }^{\mathrm{a}}$ and Peter Yeboah ${ }^{\mathrm{b}}$}

a MD, MPH, EMBA, Deputy Executive Director, Christian Health Association of Ghana (CHAG)

${ }^{b}$ M.Sc. MPH/ICHD, BSc, Executive Director, Christian Health Association of Ghana (CHAG)

\section{Abstract}

Introduction: The interphase of faith and practicing health professionally often presents a challenge. To navigate between the two requires tact, experience, and professionalism. Such is the case of the Christian Health Association of Ghana (CHAG).

Objective: This case report presents an overview of how the Christian Health Association has provided family planning services for marginalized communities in Ghana by Church denominations some of whose doctrines forbid them from practicing certain forms of family planning.

Background: $\mathrm{CHAG}$ is a network of 300 health facilities and health training institutions owned by 25 different Christian church denominations. CHAG provides health care to the vulnerable, deprived, and marginalized population groups in all 10 regions of Ghana. As an implementing partner of the Ministry of Health, CHAG is mandated to implement key policies to achieve national health outcomes, including family planning. Some members do not accept artificial family planning on the account of religious faith. This often presents a challenge in addressing the need to provide family planning services as required. This challenge is further complicated by the fact that the CHAG secretariat charged with protecting the interests of member institutions as well as those of the ministry.

Strategy: $\mathrm{CHAG}$ tailors the provision of family planning interventions to denominational, socio-cultural, and religious acceptability, and client needs.

Results: Over a period of three years, the proportion of family planning acceptors who are adolescents (10-19 years) increased from 13.7 percent to 17.0 percent, total family planning acceptors increased from 67,312 to 73,648 and total couple years of protection increased from 71,296 to 92,852 . Male sterilization also increased, contrary to cultural beliefs. Importantly, CHAG as an implementing partner is able to satisfy its obligation of providing service to achieve national health goals.

Conclusion: Protecting the interests of Christian health institutions and those of government may conflict at some point. Being tactful and allowing work within the confines of faith and obligations helps in achieving desired results. 


\section{Introduction}

Family planning refers to a conscious effort by a couple to limit or space the number of children they have through the use of contraceptive methods. Family planning services are therefore services designed to help couples and individuals of reproductive age (15-49 years) to space their children, prevent unwanted pregnancies, and improve reproductive health. There is evidence to show that family planning improves health, reduces poverty, and empowers women. ${ }^{1}$

In developing countries such as Ghana, there are many impediments to family planning including limited access to services, products, and information; lack of spousal consent; and, in particular, issues of faith and religion. Christian health organizations take interest in faith and practice. The interface between faith and practicing health professionally often presents a challenge. To navigate between the two requires tact, experience, and professionalism. Such is the case of the Christian Health Association of Ghana (CHAG). This case report presents an overview of how 25 denominations (the Association) have institutionalized the provision of family planning services, some of whose doctrines prohibit the use of certain forms of family planning.

According to the 2014 Ghana Demographic and Health Survey, knowledge of contraception is universal in Ghana. ${ }^{2}$ Twenty-seven percent of currently married women use contraception; 22 percent use a modern method. The three most popular modern methods used by married women are injectables ( 8 percent), implants ( 5 percent), and pills ( 5 percent). Use of modern methods has more than quadrupled in the past 25 years, rising from 5 percent in 1988 to 22 percent in 2014. The government sector remains the major source of contraceptives in Ghana, providing them for 64 percent of current users, an increase from 39 percent in 2008. Overall, one in four contraceptive users discontinues using a method within 12 months of starting its use. Six percent of episodes of discontinuation occur because of side effects or health concerns. Thirty percent of currently married women have an unmet need for family planning services, meaning they are fertile and desire to either stop or postpone childbearing but are not currently using a contraceptive method, ${ }^{3}$ with 17 percent having an unmet need for spacing and 13 percent having an unmet need for limiting. ${ }^{2}$

The government of Ghana believes that Ghana's population dynamics can be turned into a valuable demographic dividend only if investments are made in family planning and reproductive health programmes to promote a lower fertility rate and more balanced age structure, as well as investments in multi-sectoral social and development programs that enable economic growth and quality-of-life improvements. ${ }^{4}$ Consequently, the objectives of Ghana's family planning policy, called Ghana Family Planning Costed Implementation Plan (GFPCIP), for 2016-2020 fiscal year are:

1) increase the modern contraceptive prevalence rate (CPR) amongst currently married women by 33 percent by 2020 (base CPR in 2014 was 22.2 percent) and

2) increase the modern CPR amongst unmarried, sexually active women from 31.7 percent in 2014 to 40 percent by 2020 .

The key areas being addressed in the GFPCIP include demand creation, service delivery, contraceptive security, policy and enabling environment, financing, stewardship, management, and accountability. These national objectives and priority areas are to be implemented by all agencies of the Ministry of Health $(\mathrm{MOH})$, both public and private, including CHAG.

As a network of 300 health facilities and training institutions, CHAG is composed of 25 different church denominations and provides health care to the most vulnerable, deprived, marginalized, and underprivileged population groups in all 10 regions of Ghana, particularly in the most remote areas. These denominations include Catholic (44 percent), Presbyterian (18 percent), Adventist (11 percent), Methodist (8 percent), Anglican (3.5 percent), Pentecostal (3.2 percent), and other (12.3 percent). Serving in 177 districts, CHAG provides curative, 
preventive, and rehabilitative care to about 6 million Ghanaians every year. As an implementing partner of the $\mathrm{MOH}, \mathrm{CHAG}$ is mandated to implement key policies to achieve national health outcomes. Such policies include family planning, maternal, and child health services.

\section{The challenge}

There are two situations that conflict in administering family planning services within the CHAG network. First, one of the core functions of CHAG is to protect and promote the interest of its member institutions. Second, as an implementing partner and an agency of the $\mathrm{MOH}, \mathrm{CHAG}$ is required to provide all services approved by the ministry including family planning. The Catholics, who form about 44 percent of the CHAG membership, do not accept artificial family planning, which is one of the main forms of family planning. The remaining 56 percent of members, including Pentecostals, do not have doctrines against the utilization and provision of family planning services. The challenge is thus to provide a service required by the $\mathrm{MOH}$ and at the same time to protect the interest of the members. Until 2013, the Catholics formed more than 70 percent of the CHAG network and, given their majority status and doctrine, family planning did not feature prominently in CHAG's list of priorities. After 2013, about 111 new members were added, the majority of whom were not Catholic institutions and who had no doctrinal challenges with providing artificial family planning services.

Objective 5 of Ghana's 2014-2017 Health Sector Medium Term Development Plan (HSMTDP) sought to enhance national capacity for the attainment of health-related Millennium Development Goals (MDGs). ${ }^{5}$ Through this objective, Ghana's health sector working group (a group of representatives of the $\mathrm{MOH}$ implementing agencies and development partners in health) agreed that all agencies implementing the MDG accelerated framework should train service providers in artificial family planning methods, particularly implant insertion. Consequently, family planning was prioritized by all agencies, including $\mathrm{CHAG}$, such that about 115 midwives and community health nurses were trained in family planning methods within CHAG.

\section{How CHAG resolves conflicts in administering family planning}

Of the various forms of family planning, the artificial forms, including oral contraceptives, barrier methods, injectables, and implants, have proven to be highly effective and easy to use. The Government of Ghana's commitment to reach more women, youth, and adolescents and make services affordable, accessible, and equitable for all population groups involves promoting the artificial methods. However, not all the populations served by CHAG accept the artificial methods even when highly promoted. Specifically the Catholics do not subscribe to the artificial and modern forms of conception due to doctrinal concerns. The Presbyterians, Adventists, Methodists, Anglicans, and the other minor groups who form 18 percent, 11 percent, 8 percent, and 19 percent respectively do not have any doctrinal inhibitions to family planning utilization and service provision. All the denominations accept single marriages, and so marriage does not usually pose restrictions to family planning within the network. Aside from the doctrinal barriers for the Catholics, there are also cultural barriers to providing modern family planning services in certain communities where CHAG operates, specifically beliefs that women might become unfaithful if they accept family planning services.

To deal with these situations while protecting and promoting the interest of its members, CHAG's strategies for family planning services are tailored, ensuring that interventions within specific denominations are socio-culturally and religiously acceptable by both the denomination and the community for whom they are intended. For example, since the Catholics do not accept artificial family planning, training programs on implant insertion exclude Catholic health institutions. Likewise, artificial family planning supplies like oral contraceptives, implants, and condoms are given only to facilities 
that accept these methods. The Regional Health Administration offices of the Ghana Health Service, through their District Health Management Administration (DMHA), provides family planning supplies every month to all health facilities in their catchment districts, including CHAG facilities. These supplies are supported by donor partners including United Nations Populations Fund (UNFPA). Facilities that believe only in natural family planning would only be given education on natural family planning and supplies like CycleBeads ${ }^{\circledR}$, which are used to plan or prevent pregnancy by tracking the start dates of a woman's period based on the Standard Days Method (SDM). CHAG member institutions that do not accept artificial family planning methods have agreed to refer clients requiring artificial family planning to the nearest facility that offers those services (a government-, CHAG-, or Marie Stopes-accredited facility).

Cultural barriers are dealt with differently by different church denominations. For example, communities that do not accept injectables are not forced to have them. Voluntary acceptance is encouraged. Often, innovative ways of dealing with such cultural barriers have been explored by members. For the most part, counseling for both members of a couple is conducted together, particularly in Northern Ghana where decisions are primarily made by men. CHAG, through its various denominations, uses quarterly community durbars - when education on pertinent health issues, including the need for family planning, is conducted - to address cultural barriers, along with conversations with traditional and opinion leaders.

CHAG implements both artificial and natural family planning methods. The natural methods that are provided include rhythm, CycleBeads ${ }^{\circledR}$ (SDM), lactation amenorrhoea (LAM), etc. Natural family planning is considered one of the oldest methods women have used to control their fertility, and addresses the concerns of a diverse population of Ghanaians with varying religious and ethical needs. Modern family planning services include injectables and voluntary surgical interventions (sterilizations) for both men and women.
A majority of people view these modern methods as more effective than natural methods. Consequently, there is the tendency for practitioners (doctors, midwives, and community health nurses) to promote modern methods, particularly injectables. A common injectable used is medroxyprogesterone (Depo-Provera) and often midwives tend to promote it particularly among the youth. This is evident in the kind of advertisements and family planning educational programs that are run on national television channels. Rarely would one find natural family planning being promoted. The excessive focus on artificial family planning creates pressure to utilize these methods, which are often not accepted by certain cultures and certain religious views. This leads to resistance and low acceptance. Prior to 2012, CHAG did not have any significant data on family planning but the need to track the effectiveness of their approach was needed..

\section{Methods}

Data for this study was taken from routine service data collected from member institutions by the Health Management Information Systems (HMIS) unit of CHAG. This is part of quarterly data that member institutions submit to the HMIS for policy, planning, and decision-making. The same data is submitted to the District Health Information Management Systems 2 (DHIMS2) software of the Ghana Health Service. Data collected include outpatient and inpatient service utilization, morbidity and mortality for all age groups, and family planning service (various methods) utilization, and is submitted using the $\mathrm{MOH}$ service reporting formats. Other data that is routinely collected by the HMIS unit include antenatal and postnatal care utilization data, supervised and non-supervised deliveries, immunizations, outreach services, diseases of public health concern, and human resources. The data is Excel-based and is stored on a server at the CHAG secretariat's HMIS unit.

For this study, data on family planning from the various institutions were collated from 2013-2015 and were based on 93 percent response rate. Data 
was analyzed using Excel for computations of averages, percentages, and total numbers. Analyzed data was compared with data reported in the DHIMS to ensure reliability and, where necessary, facilities were called for clarification. Results from the analysis were reported in tabular forms based on which interpretations were made. Other information used in this study was taken from CHAG's 2013-2015 annual reports.

\section{Results}

Broadly, all the interventions that CHAG implements are within the national priority areas, thus satisfying the requirements for the health sector for which CHAG is mandated. Secondly, implementing strategies that are within the confines of the beliefs and faith satisfies CHAG's stewardship mandate. Largely, because this approach is socio-culturally and religiously acceptable, patronage and prevalence of contraception is appreciable. In 2015, a total of 15,101 people accepted natural family planning, which formed about 20.5 percent of total family planning acceptors. The number that accepted natural family planning in 2015 was 4,757 (46 percent) more than 2014. Of the acceptors of natural family planning for 2015, a total of 13,881 (91.9 percent) used LAM whilst 1,220 (8.1 percent) used SDM. Though the proportion of persons using natural family planning is small, CHAG is assured that all populations, no matter how small, are offered care. Importantly, CHAG has observed a significant increase in the proportion of family planning acceptors who are adolescents, an indication of the fact that cultural barriers to adolescents using family planning are being reduced. Over a period of three years (2013-2015), the proportion of family planning 2013-2015 acceptors who were adolescents (10-19 years old) increased from 13.7 percent to 17.0 percent, the total number of family planning acceptors increased from 67,312 to 73,648 (a 9.4 percent increase) and total couple years of protection rose from 71,296 to 92,852 (a 30.2 percent increase). ${ }^{6}$ Table 1 shows the trend of family planning acceptors in CHAG facilities and couple years of protection from 20132015. Male sterilization also increased, contrary to cultural beliefs. In 2013, there was no vasectomy performed in any of the numerous CHAG facilities. In 2014 and 2015, the number of vasectomies recorded were 4 and 14, respectively, whilst female sterilization increased from 455 in 2014 to 807 (a 77.4 percent increase) in 2015 , as seen in Table $2 .{ }^{6}$ The increased female sterilization is in tandem with growing trends of Caesarean Section $(\mathrm{C} / \mathrm{S})$ rates observed within the CHAG network. Between 2012 and 2016, the C/S rate has increased by 43 percent and this may partly account for the rising female sterilization. ${ }^{7}$ Generally, family planning utilization increases as the number of children increases. ${ }^{8}$ When women have more than three deliveries by $\mathrm{C} / \mathrm{S}$, they are often counseled on medical grounds to undergo sterilization. Intensive education on family planning

Table 1. Family planning acceptors and couple years of protection,

\begin{tabular}{|l|c|c|c|}
\hline \multicolumn{1}{|c|}{ Description } & 2013 & 2014 & 2015 \\
\hline $\begin{array}{l}\text { Percentage postnatal registrants accepting family } \\
\text { planning }\end{array}$ & 16.7 & 14.6 & 14.8 \\
\hline $\begin{array}{l}\text { Proportion of FP acceptors who were } \\
\text { adolescents (10-19) }\end{array}$ & 13.7 & 15.0 & 17.0 \\
\hline $\begin{array}{l}\text { Proportion of FP acceptors who were older } \\
\text { adolescents (15-19) }\end{array}$ & 12.3 & 14.2 & 16.0 \\
\hline Total family planning continuing acceptors & 44,668 & 45,526 & 50,293 \\
\hline Total family planning new acceptors & 22,644 & 21,969 & 23,355 \\
\hline Total drop-outs & - & 21,786 & 17,202 \\
\hline Total family planning acceptors & 67,312 & 67,495 & 73,648 \\
\hline Total couple years protection & 71,296 & 69,701 & 92,852 \\
\hline
\end{tabular}


Table 2. Trend of family planning by type, 2013-2015

\begin{tabular}{|l|c|c|c|}
\hline \multicolumn{1}{|c|}{ Description } & 2013 & 2014 & 2015 \\
\hline Natural family planning & 10,821 & 10,344 & 15,101 \\
\hline Male sterilization (vasectomy) & 0 & 4 & 14 \\
\hline Female sterilization & 463 & 455 & 807 \\
\hline Condom (male) & 3,748 & 4,591 & 8,192 \\
\hline Condom (female) & 81 & 162 & 108 \\
\hline Oral contraceptives (the pill) & 10,542 & 11,592 & 10,810 \\
\hline Implant & 2,956 & 3,308 & 4,336 \\
\hline $\begin{array}{l}\text { Short term injectable (depot } \\
\text { medroxyprogesterone acetate) }\end{array}$ & 36,870 & 32,799 & 33,947 \\
\hline $\begin{array}{l}\text { Intra-uterine contraceptive device } \\
\text { (IUCD) }\end{array}$ & 119 & 134 & 202 \\
\hline All other artificial methods & 64 & 93 & 131 \\
\hline
\end{tabular}

is another factor that may have played a key role in the increased trends observed in both male and female sterilizations.

In $2015,73,648$ postnatal women (14.8 percent) accepted various forms of family planning. ${ }^{6}$ This represents an increase from 67,312 in 2013 to 73,648 (9.4 percent) in 2015 as shown in Table 1. Table 2 gives a trend of the various family planning methods from 2013 to 2015. Consistent with findings from research conducted by Amu and Nyarko, ${ }^{9}$ it is to be noted that the short-term injectable (Depot Medroxyprogesterone acetate) is the most accepted contraceptive within the CHAG network.

\section{Discussion}

Faith and cultural beliefs must be linked to the knowledge of family planning in order to increase family planning uptake. Implementing interventions that are socially, culturally, and religiously acceptable are likely to yield appreciable outcomes. There is an increasing trend of using family planning methods within the CHAG network, and this may be related to the CHAG's program and observed national trends. At the national level, the last Ghana Demographic and Health Survey indicated that from 2008 to 2014 , the use of any method of contraception increased from 24 percent to 27 percent and modern methods had increased from 17 percent to 22 percent. Use of family planning increases with the prevalence of outreach campaigns. ${ }^{8}$ Thus, education on family planning, increased advertisements, and stakeholder engagements on family planning contributed to the increasing trends of family planning acceptance. The increased proportion of adolescents using family planning may be an indication of the fact that cultural barriers to adolescents using family planning are being reduced.

CHAG data also shows that 13,881 family planning acceptors (18.9 percent) use LAM. ${ }^{6}$ This contrasts with national findings where only 0.2 percent of married women use LAM. ${ }^{8}$ But this situation draws attention to the need for complementary services between governments and faith-based health systems in achieving national health outcomes. Where each has a deficiency, the other can fill in the gap, as in the case of LAM in Ghana. Being religious, cultural, and client sensitive may lead to increased LAM acceptors and proportions of adolescents who use family planning methods. Additionally, male and female sterilization could increase when family planning practitioners are culturally sensitive.

Over the years, implementation of interventions and policies has typically been a top-down approach where agencies are expected to accept and implement policies as they are. By taking a bottom-up approach that responds to faith and culture, contraceptive use is increased. A top-down approach often presents a challenge. Though implementation may 


\section{Duah and Yeboah}

be done, it may not yield the impact it should since it may not be socio-culturally acceptable.

Denominations may not accept certain forms of family planning but can easily refer clients who require those services to facilities that do provide them. This allows faith-based organizations to satisfy clients' needs and policy requirements for service provision whilst remaining true to their doctrinal beliefs.

The limitations of this study are that it may not be generalizable, and for that matter one cannot be sure how this would work in another context. CHAG's approach correlated with improved family planning acceptance, but this does not mean it caused it. There

\section{Christian Journal for Global Health |}

was no comparison group; indeed a comparison study may not be feasible. Like any other case report study, the findings in this study may be over-interpreted, and readers may be distracted when focusing on the unusual. More research is required with other Christian health service organizations with similar socio-cultural contexts.

\section{Conclusions}

The interests of Christian health facilities and those of the government may conflict at some point. Being tactful and allowing work within the confines of faith and obligations helps in achieving desired outcomes.

\section{References}

1 Bongaarts J, Cleland J, Townsend WJ, Bertrand TJ Gupta DM. Family planning programs for the 21 st century: rationale and design. New York: Population Council; 2012

2 Ghana Statistical Service. Ghana Demographic and Health Survey 2014. Rockville, Maryland, USA. April 29, 2016

3 MEASURE Evaluation project [Internet]. Unmet need for family planning. [updated June 4, 2016] Available at: https://www.measureevaluation.org/prh/rh_indicators/ specific/fp/unmet-need-for-family-planning

4 Government of Ghana. Ghana Family Planning Costed Implementation Plan. Washington D.C., Ghana: Futures Group, Health Policy Project. April 26, 2015

5 Ministry of Health. (2014). The Health Sector Medium Term Development Plan. Accra: Powerhaus Media Concepts.

Peer Reviewed

Competing Interests: None declared.
6 CHAG. (2013-2015). Reports from Minimum Data Set. Accra: $\mathrm{CHAG}$

7 Christian Health Association of Ghana. Annual Report 2016. Accra, Ghana. May 31, 2017

8 Ghana Statistical Service. (2014). Ghana Demographic and Health Survey. Accra: Ghana Health Service

9 Amu H, Nyarko SH. Trends in contraceptive practices among women in reproductive age at a health facility in Ghana: 2011-2013. Contraception and Reproductive Medicine. 2016; 1:2;1-5. https://doi.org/10.1186/s40834016-0010-9

Correspondence: James Duah, james.duah@chag.org.gh; Peter Yeboah, peter.yeboah@chag.org.gh

Cite this article as: Duah J and Yeboah P. Family planning practice among Christian health service providers in Ghana: a case study. Christian Journal for Global Health. July 2017; 4(2):80-86; https://doi.org/10.15566/cjgh.v4i2.175.

(C) Duah J. and Yeboah P. This is an open-access article distributed under the terms of the Creative Commons Attribution License, which permits unrestricted use, distribution, and reproduction in any medium, provided the original author and source are properly cited. To view a copy of the license, visit http://creativecommons.org/licenses/by/4.0/ 